\title{
How supercooled liquid phase-change materials crystallize: Snapshots after femtosecond optical excitation
}

\author{
Peter Zalden ${ }^{1,2}$, Alexander von Hoegen ${ }^{3}$, Patrick Landreman ${ }^{5}$, Matthias Wuttig ${ }^{3,4}$ and Aaron M. \\ Lindenberg ${ }^{1,2,5}$ \\ 1) Stanford Institute for Materials and Energy Sciences, SLAC National Accelerator Laboratory, Menlo Park, CA 94025, USA \\ 2) PULSE Institute, SLAC National Accelerator Laboratory, Menlo Park, CA 94025, USA \\ 3) I. Physikalisches Institut (IA), RWTH Aachen University, 52056 Aachen, Germany \\ 4) JARA-Fundamentals of Information Technology, RWTH Aachen University, 52056 Aachen, Germany \\ 5) Department of Materials Science and Engineering, Stanford University, Stanford, CA 94305, USA
}

\section{Keywords}

chalcogenide glasses; kinetic fragility; crystal growth; phase-change materials; glass formation

\begin{abstract}
Glass forming materials are employed in information storage technologies making use of the transition between a disordered (amorphous) and an ordered (crystalline) state. With increasing temperature the crystal growth velocity of these phase-change materials becomes so fast that prior studies have not been able to resolve these crystallization dynamics. However, crystallization is the time limiting factor in the write speed of phase-change memory devices. Here, for the first time, we quantify crystal growth velocities up to the melting point by using the relaxation of photo-excited carriers as an ultrafast heating mechanism. During repetitive femtosecond optical excitation, each pulse enables dynamical evolution for tens of picoseconds before the intermediate atomic structure is frozen-in as the sample rapidly cools. We apply this technique to $\mathrm{Ag}_{4} \mathrm{In}_{3} \mathrm{Sb}_{67} \mathrm{Te}_{26}$ (AIST) and compare the dynamics of as-deposited and application-relevant melt-quenched glass. Both glasses retain their different kinetics even in the supercooled liquid state, thereby revealing differences in their kinetic fragilities. This approach enables the characterization of application-relevant properties of phase-change materials up to the melting temperature, which has not been possible before.
\end{abstract}

Mankind has utilized glasses during the last five thousand years. They can be prepared by cooling a liquid fast and far enough below the glass transition temperature - to a temperature where its viscosity is sufficiently high that the atomic arrangement is kinetically frozen-in ${ }^{1}$. Until recently, research and technology have focused on good glass formers, i.e. materials which can be vitrified by cooling their liquid state at moderate rates. But in recent decades poor glass formers such as metallic glasses and certain chalcogenide glasses have gained interest due to their remarkable property portfolio ${ }^{2,3}$. These materials need to be cooled at rates in excess of around $3 * 10^{9} \mathrm{~K} / \mathrm{s}$ to bypass crystallization and to quench the atoms in an amorphous arrangement ${ }^{4}$. This so-called glass transition at temperature $T_{g}$ is commonly observed at a 
viscosity close to $10^{12} \mathrm{~Pa} \mathrm{~s}^{5,1}$, where the change in viscosity with reciprocal temperature is quantified by the fragility

$$
m=\left.\frac{\partial \log \eta}{\partial\left(T_{g} / T\right)}\right|_{T=T_{g}}
$$

A material whose supercooled liquid has a high fragility is better suited for applications where the timescale of atomic dynamics needs to change over small temperature ranges. One such example are phase-change materials (PCMs) for non-volatile data storage ${ }^{6}$, where information is encoded in the amorphous and crystalline state of individual volumes. These PCMs need to crystallize rapidly when an external stimulus provides heat ${ }^{7}$, but at the same time have to retain their amorphous state over tens of years under ambient conditions. Therefore, a pronounced temperature dependence of their viscosity is crucial for their application. The write speed of these memory devices is currently limited by the time it takes to crystallize the active material with crystal growth velocities that approach the speed of sound ${ }^{8}$, rendering an experimental determination challenging. An acoustic phonon that dominates diffusive transport of heat cannot propagate faster than the speed of sound and therefore it is not surprising that at highest temperatures a glassy PCM on a heating stage cannot equilibrate before crystallization sets $i^{9}$. Earlier studies in the low-temperature regime have determined crystal growth velocities of as-deposited (AD) $\mathrm{PCMs}^{10}$ and a comparison with growth velocities of melt-quenched (MQ) material ${ }^{11}$ revealed a few hundred times higher rates in the MQ glassy state. Laser-driven experiments revealed that shorter pulses can induce crystallization in the MQ but not in the AD state ${ }^{12,13}$. However, approaches to study crystal growth velocities based on optical lasers or electrical excitation were limited to pulse lengths longer than a nanosecond and therefore could not determine growth velocities over the entire range of temperatures ${ }^{11,13-16}$. Ultrashort laser pulses have also been employed to excite PCMs, but only the amorphization process was studied systematically as a function of fluence ${ }^{17,18}$. Here we demonstrate how ultrashort laser pulses can be employed to study the crystallization of PCMs and at the same time overcome earlier limitations on the heating rates. Optical excitation with femtosecond laser pulses heats the lattice through relaxation of photoexcited carriers on the picosecond timescale ${ }^{19}$ and corresponds to heating rates around $3^{*} 10^{14} \mathrm{~K} / \mathrm{s}$. Using the optical reflectance as probe we find that repetitive excitation with these pulses can drive the crystallization process and by changing their fluence, the crystal growth conditions can be chosen. With this approach we are able to access the full temperature range of the crystallization process including the full supercooled liquid regime of as-deposited (AD) and melt-quenched (MQ) PCMs. 


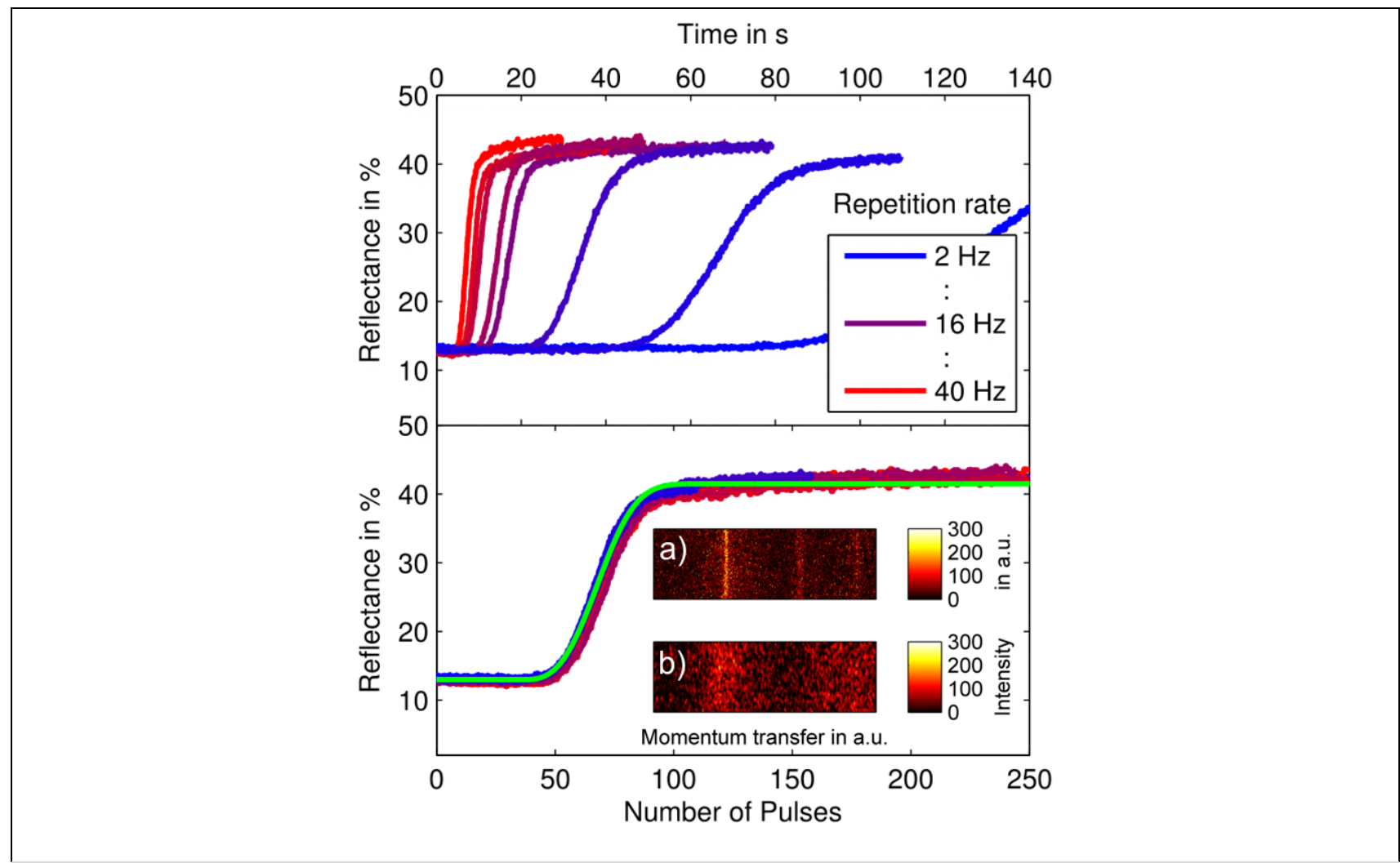

Fig. 1: Reflectance traces at $633 \mathrm{~nm}$ were recorded while exposing a thin film of as-deposited AIST to fspulses at various repetition rates (red to blue curves, upper panel). All traces come to congruence if plotted as a function of the number of pulses applied and match the sigmoidal shape of the JMAK model (green curve, lower panel). Insets $a$ ) and $b$ ) depict the raw diffraction patterns for the final crystalline and initial amorphous state, respectively (discussed in detail in Suppl. Sec. 7).

In this study we focus on the PCM Ag $4{ }_{4} \mathrm{n}_{3} \mathrm{Sb}_{67} \mathrm{Te}_{26}$ (AIST), commonly used in optical data storage ${ }^{2,11}$. Exciting a thin film of this material repetitively with ultrashort optical pulses below the melting threshold triggers a continuous increase in reflectance as shown in Fig. 1 for different repetition rates of the pump laser. Diffraction data shown as an inset in Fig. 1 provide evidence that this increase in reflectance is due to the crystallization of AIST. Most interestingly a transformation of the time axis to the individual number of pulses applied brings all curves to congruence, collapsing them onto a single trajectory. For all repetition rates at and below $40 \mathrm{~Hz}$, each pulse of this specific fluence has the same effect on the transformation process - independent of the time delay to the previous pulse. Each pulse induces an irreversible modification in the amorphous sample that contributes to the overall crystallization process, even if there is no obvious change in the optical reflectance. The initial absence of a modification of optical parameters might be related to both, incubation and nucleation effects as well as early growth of nuclei that already exist in the as-deposited ${ }^{20}$ or melt-quenched glass. This technique allows studying the crystal nucleation and growth in a glass in unprecedented detail, because each intermediate stage of the crystallization 
process can be investigated for whatever time the respective probe requires (e.g. by $\operatorname{TEM}^{20}$ or $\mathrm{AFM}^{10}$ ). These frozen-in intermediate states can contain both sub-critical as well as over-critical nuclei. Each optical pulse has $350 \mathrm{fs}$ duration and irreversibly changes the atomic arrangement in an accumulative way (see supplement Sec. 6 for a discussion on pulse duration).
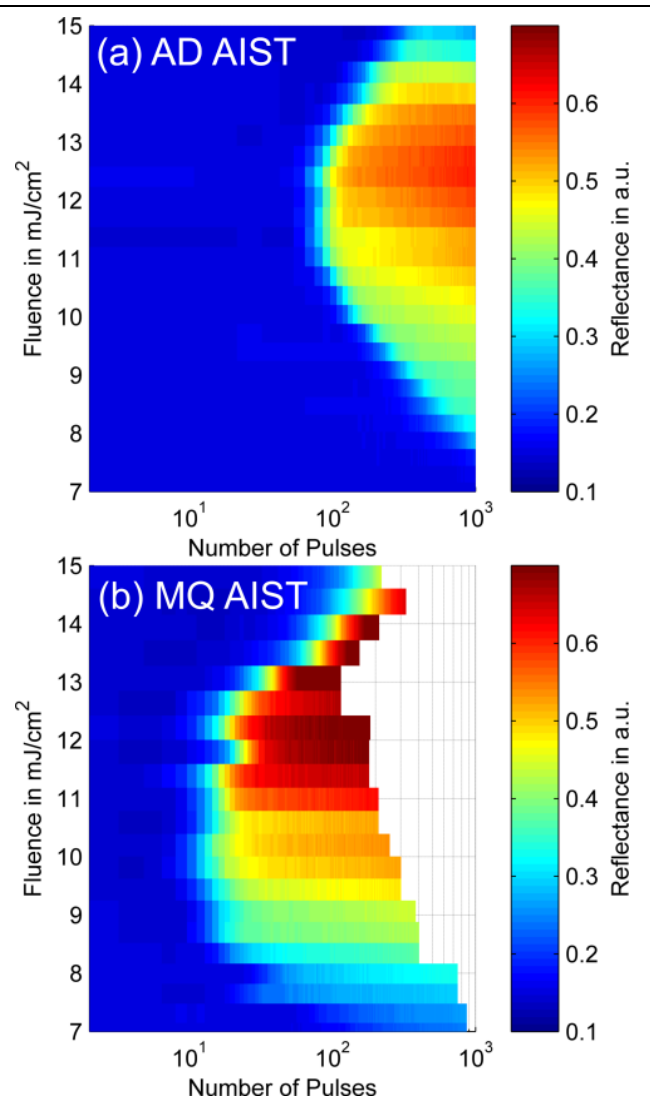

Fig. 2: Reflectance traces of (a) as-deposited (AD) and (b) melt-quenched (MQ) AIST on the same scale: Crystallization requires far fewer pulses for the MQ glass, which therefore crystallizes more rapidly. The MQ glass is obtained after initiating a crystallized film of equal thickness with a single pulse well above the melting threshold $\left(32 \mathrm{~mJ} / \mathrm{cm}^{2}\right)$.

We now turn to discussing the fluence dependence of the reflectance traces of AIST in both as-deposited $(A D)$ and melt-quenched (MQ) glasses as a means of investigating the temperature dependence of the crystallization process. Fig. 2 shows the reflectance traces after repetitive excitation of the PCM under various fluences as a function of the number of pulses applied. The data on AD glass were obtained upon excitation with $40 \mathrm{~Hz}$ repetition rate femtosecond pulses, while probing the reflectance with $\mathrm{CW}$ white light through a microscope objective. The probe spot size was $8 \mu \mathrm{m}$ in diameter and much smaller than the pump spot with Gaussian profile and 1/e-diameter of $280 \pm 20 \mu \mathrm{m}$. Under these conditions, the probe area is homogeneously excited by each laser pulse. The same measurements, except for a lower repetition rate of $4 \mathrm{~Hz}$ were performed on $\mathrm{MQ}$ samples, starting from crystallized films and using a pre-pulse of the same 
spot size but higher pulse energy $\left(32 \mathrm{~mJ} / \mathrm{cm}^{2}\right)$ to melt-quench the PCM. Already the raw reflectance traces in Fig. 2 reveal important insight into the crystallization behavior of AIST; (i) The MQ glass requires less than a tenth the number of pulses to crystallize than the $A D$ state even up to excitation conditions where the material starts to melt; (ii) The crystallization speed reaches a maximum at some fluence significantly below the melting threshold.

The data shown in Fig. 2 can be transformed into quantitative information on crystal growth using two transformations. First, the fluence is linearly transformed to an effective temperature $T^{\prime}$ with thermal properties being roughly constant over the investigated temperature range of $500 \mathrm{~K}$ to $800 \mathrm{~K}$, i.e. at least a factor of two above the Debye temperature $\left(179 \mathrm{~K}\right.$ for $\left.\mathrm{Sb}_{2} \mathrm{Te}_{3}{ }^{21}\right)$. We present further justification for this linear re-scaling by comparing the simulated crystal growth under repetitive excitation and under isothermal conditions based on the same model for crystal growth. The linear crystal growth velocity is given by ${ }^{11}$

$$
u(T)=\frac{8 D(T) r_{\mathrm{at}}^{2}}{\lambda^{2} R_{h}}\left[1-\exp \left(-\frac{\Delta G(T)}{k_{B} T}\right)\right]
$$

where $\lambda$ is the diffusional jump distance, $R_{h}$ is the hydrodynamic radius and $r_{\mathrm{at}}$ the radius of an average atom. The diffusivity $D(T)$ is approximated by the Stokes-Einstein equation ${ }^{22}$ and the temperature dependence of viscosity described by the MYEGA model ${ }^{23}$. It is worth mentioning that the Stokes-Einstein equation has been shown to be valid during supercooling up to $300 \mathrm{~K}$ below the melting point in the case of the PCM GeTe, but breaks down upon further cooling ${ }^{24}$. The enthalpy difference $\Delta G$ between the supercooled liquid and the crystalline state can be interpolated ${ }^{25}$. These equations fully describe the temperature dependence of the crystal growth velocity $u(T)$ and can be evaluated for any temperature based on the parameters from measurements and estimations available from literature on AIST (see supplement Sec. 1).

Finite element simulations (FES) of the transient temperature distribution show that the PCM remains at the temperature induced by the relaxation of photo-excited carriers for several tens of picoseconds. It is expected that due to the super-exponential scaling of the growth velocity with temperature, the entire crystallization process is dominated by the highest temperatures reached - until the highest growth velocity occurs. At even higher excitation conditions, growth at the peak temperature and growth at highest velocity both contribute to the overall process. In this case, simulations are required to model the growth process under non-isothermal conditions. Our results, explained in Sec. 1 of the SI, show that the error in this temperature range between $700 \mathrm{~K}$ and the melting point at $807 \mathrm{~K}^{26}$ is below a factor of $1.2^{+/ 1}$. Therefore, $F=\alpha\left(T^{\prime}-T_{0}\right)$, where $F$ is the incoming pump fluence and $T_{0}$ denotes ambient temperature. Here $\alpha=0.0274 \mathrm{~mJ} \mathrm{~K}^{-1} \mathrm{~cm}^{-2}$ (consistent with $0.0213 \mathrm{~mJ} \mathrm{~K}^{-1} \mathrm{~cm}^{-2}$ from FES) is determined by aligning the slowing down of the transformation to the melting temperature of the crystal. It is worth mentioning that 
the optical properties of AD and MQ AIST are very similar even around the band gap (see Fig. S3 in the supplement), so that differences in the absorption and resulting temperature scale are expected to be far smaller than the less than $6 \%$ absorption change upon crystallization (see Tab. S1). From the simulations of crystal growth in Sec. 1 of the supplement we see that every single pulse induces $\tau^{*}=19$ ps of effective crystal growth before the material kinetically freezes in. A rough confirmation of this number can be obtained as follows: Multiplying the lowest number of pulses required to obtain full crystallization ( 10$)$ with the maximum growth velocity estimated ${ }^{11}, 100 \mathrm{~m} / \mathrm{s}$, and the effective growth time per pulse, $19 \mathrm{ps}$, one obtains a grain radius of $19 \mathrm{~nm}$. This number is in excellent agreement with the final grain radius of $19 \mathrm{~nm}$ derived from powder diffraction data ${ }^{27}$ using the Scherrer equation.

The second conversion involves the transformation of reflectance changes to the speed of crystal growth. The data in Fig. 2 show that the first pulses do not have a noticeable effect on reflectance. We associate this delay with a combination of incubation, nucleation and early growth processes. Note that even after reaching the critical nucleus size of 33 atoms in $\mathrm{AIST}^{28}$, the volume of a critical nucleus $\left(1 \mathrm{~nm}^{3}\right.$ for an atomic number density of 0.0308 atoms $/ \AA^{329}$ ) is far smaller than the volume of a final crystallite, $\beta^{-1}=28700 \mathrm{~nm}^{3}$, obtained from the Scherrer equation. We therefore assume that the increase in reflectance originates entirely from the linear growth of nuclei far above criticality, for which the JMAK model as derived e.g. by Kolmogorov can be applied ${ }^{30}$. Then, the volume fraction occupied by grains at time $t$ after the growth was initiated at time $t_{0}$ is given by

$$
x(t)=1-\exp \left(-\frac{4 \pi}{3}\left(u\left(t-t_{0}\right)\right)^{3} \beta\right) \quad \text { for } \quad t>t_{0}
$$

where $u$ is the velocity of linear growth and $\beta$ is the number density of nucleation centers, assumed to equal the final density of grains extracted from diffraction data. The underlying assumption is that nucleation precedes crystal growth ${ }^{9}$. The time delay $t_{0}$ represents the effects of incubation and nucleation not covered by the JMAK model. A model for three dimensional growth is chosen for two reasons: (i) the film is thicker than the average radius of crystalline grains determined by powder diffraction and (ii) it can more closely resemble the present sigmoidal shape than a model for two-dimensional growth (see refined curve in Fig. 1). In our case we express the volume transformed as $\left(u\left(t-t_{0}\right)\right)^{3}=\left(d\left(n-n_{0}\right)\right)^{3}$, where $n$ is the number of applied pulses and $d$ is the growth distance per pulse. Following this model, the crystal growth velocity $u$ can be directly extracted from the slope of reflectance traces at the point of inflection (see supplement Sec. 2). 


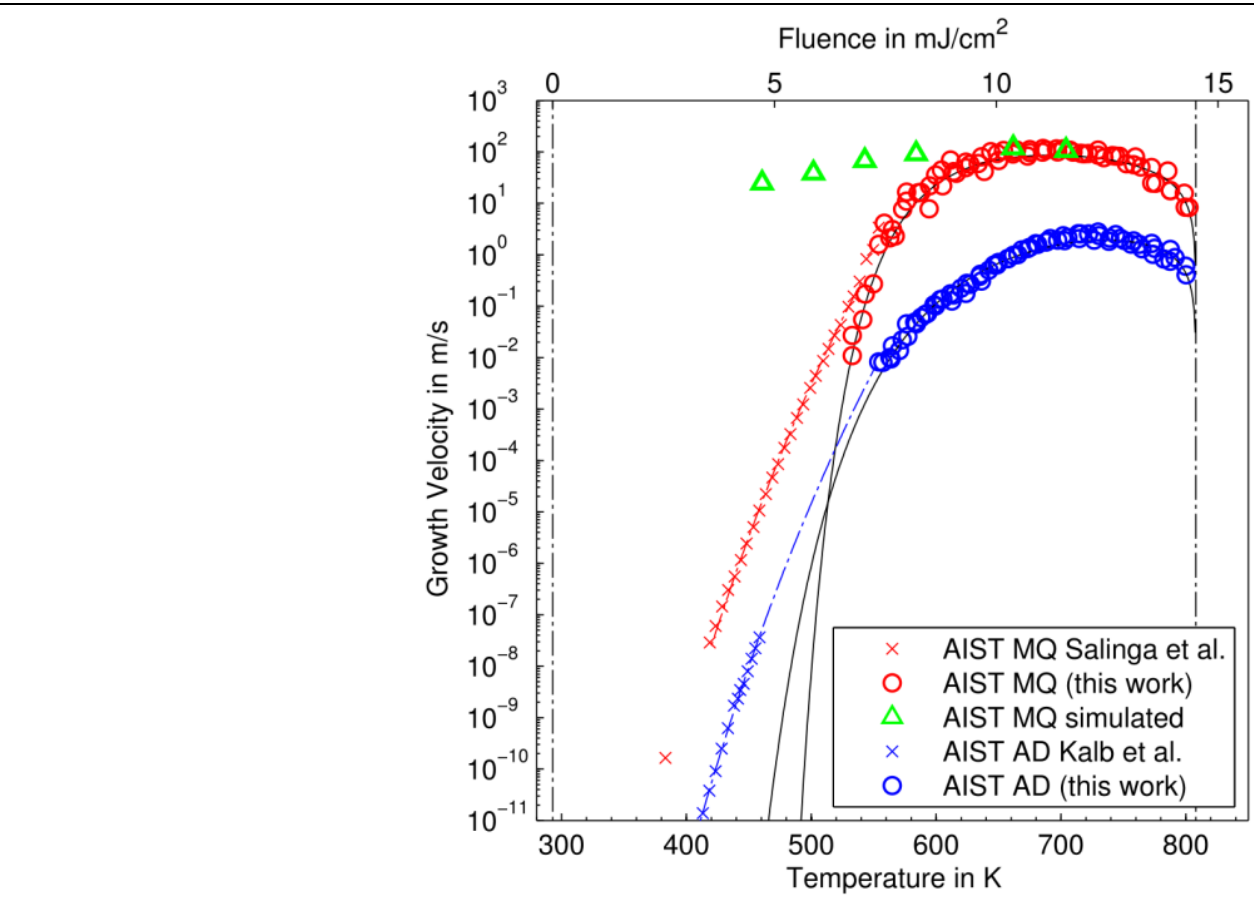

Fig. 3: Crystal growth velocities of $A D$ and MQ AIST (circles) line up well with earlier reports of measurements at lower temperatures (crosses) ${ }^{10,11}$. Most surprisingly, the two glasses crystallize with different velocities over the entire temperature range up to the melting point of the crystal. At low temperatures, the different slopes are evidence for different fragilities. Green triangles correspond to transformed diffusivities from AIMD simulations ${ }^{31}$. Dash-dotted curve: fit to literature data using an Arrhenius model for viscosity; Solid curve: fit to the present data using the MYEGA model.

Growth velocities extracted accordingly from the data in Fig. 2 are plotted as a function of effective temperature in Fig. 3. These data reveal that the crystal growth velocities of both glasses remain distinct up to the melting point. The growth velocity of the $M Q$ glass has its maximum of $110 \mathrm{~m} / \mathrm{s}$ at $700 \pm 20 \mathrm{~K}$, whereas the $A D$ glass crystallizes at most with $2.5 \mathrm{~m} / \mathrm{s}$ at $730 \pm 12 \mathrm{~K}$. It might appear surprising that such a pronounced difference in growth velocities is retained even in the supercooled liquid state, because one might expect the memory of the preparation of the glass to be lost upon reaching the supercooled liquid phase. In the present case of AIST, this memory is not lost even close to the melting point of the crystal. This might be a consequence of the timescales at elevated temperature of about 19 ps not being sufficiently longer than the timescales associated with all possible atomic dynamics. In similar glasses phonon modes as low as $5 \mathrm{GHz}$ were observed ${ }^{32}$. These modes might prevent full equilibration of the supercooled liquid.

The different behaviors of both glasses are yet another fingerprint of the high fragility of this material. Our data can be fully described using the MYEGA model (solid curves) for viscosity ${ }^{23}$. The fragilities extracted from our experimental data for the $A D$ and $M Q$ glass are $104 \pm 10$ and $217 \pm 16$, respectively. The kinetic 
fragility determined for MQ AIST is comparable to upper limits of $175^{33}$ and $250 \pm 30^{34}$. As to the best of our knowledge this is the highest kinetic fragility ever observed for an inorganic supercooled liquid and explains how this material is particularly suitable for data storage, where the high crystal growth velocity over a wide temperature range leads to short overall crystallization times. The fragility of MQ AIST is significantly higher than the value of 130 determined previously ${ }^{11}$. This deviation is caused by the lower glass transition temperature of the $A D$ material $\left(443 \mathrm{~K}^{35}\right)$ that was included in the refinement of the growth model in this earlier study of the melt-quenched phase. The resulting glass transition temperature of AD AIST (459 K) that we determined is in rough agreement with the calorimetric value $\left(443 \mathrm{~K}^{35}\right)$. Our data further reveal that the glass transition of the MQ glass takes place at even higher temperature of $483 \mathrm{~K}$. The distinct nature of these glasses is also reflected in small differences in their optical properties (see suppl. Sec. 4) and in their Raman spectra ${ }^{36}$.

Literature data for the growth velocities of AD and MQ AIST are also included in Fig. 3. In both cases they can be described by the same growth model with an Arrhenius function for the temperature dependence of viscosity. With an extrapolation to higher temperature both sets of literature data are found to tangentially overlap our crystal growth velocities. The temperature associated with this overlap separates the lowtemperature Arrhenius behavior, typical for a glass, from the high-temperature super-exponential behavior, typical for a supercooled liquid. Both are separated by the crossover temperature $T^{*}$ that unlike the glass transition temperature $T_{g}$ does not depend on the cooling rate ${ }^{37,38}$. In literature, this transition is sometimes referred to as fragile-to-strong (FTS) transition upon cooling ${ }^{39}$. In the MQ glass, it is observed to occur at $570 \pm 15 \mathrm{~K}$ and at lower temperature for the AD sample, approximately $540 \pm 20 \mathrm{~K}$. However, the crystal growth velocity of MQ AIST seems to follow the super-exponential scaling of a supercooled liquid even below its estimated freezing-in temperature. The absence of a transition to an Arrhenius behavior in our measurement range requires further attention. A comparison with molecular dynamics simulations shows exact agreement of the peak growth velocity while the simulated data deviate towards low temperatures due to known limitations in the simulation technique ${ }^{31}$. The results of our study further reveal a mechanism with which sub-ns laser-induced crystallization could be achieved. Following the observation that ten pulses with a total effective time of 190 ps fully crystallize the material we can speculate that a sequence of closely spaced pulses that maintains isothermal conditions could crystallize MQ AIST on a few hundred picosecond timescale. The resulting understanding of fs-induced crystallization might be particularly helpful for the application of PCMs as active optics ${ }^{40-42}$, which can be operated significantly faster after initiating the PCM accordingly. Similar effects have been motivated by the realization of neuromorphic computation using sub-threshold electrical pulses to initiate phase-change devices with pulses of $10 \mathrm{~ns}$ duration ${ }^{43}$. This initialization with a limited number of effectively shortens the 
remaining crystallization process, explaining what has been demonstrated using pre-pulses below the crystallization threshold on longer timescales ${ }^{44}$.

In conclusion, we present a new approach to resolve the maximum crystal growth velocities of a PCM for the first time. It enables the freezing-in of intermediate stages of the crystallization process with potential for further investigations of the nucleation process. It turns out that the PCM AIST crystallizes with different growth velocities over the whole temperature range with peak values of $110 \mathrm{~m} / \mathrm{s}$ for the $M Q$ and $2.5 \mathrm{~m} / \mathrm{s}$ for AD material. These differences are also reflected in the fragilities of the two supercooled liquids. This approach enables the determination of application-relevant properties of PCMs that were not accessible before.

\section{Associated Content}

Supporting Information. Contains: Simulation and comparison of pulse-induced crystal growth; Extraction of crystal growth velocities from reflectance traces; Experimental Setup and Procedures; Optical Properties of $A D$ and MQ AIST; Deviations at higher repetition rates; Pulse duration and effects of non-linear absorption; X-ray diffraction from laser crystallized spots. This material is available free of charge via the Internet at http://pubs.acs.org

\section{Author Information}

Corresponding Author: Peter Zalden, peter@zalden.de Present Address: European XFEL, Notkestraße 85, 22607 Hamburg, Germany

\section{Acknowledgements}

Research was supported by the U.S. Department of Energy, Basic Energy Sciences, Materials Sciences and Engineering Division. M.W. gratefully acknowledges support by the Deutsche Forschungsgemeinschaft through SFB 917 (Nanoswitches). P.Z. gratefully acknowledges funding by the Humboldt foundation. A.v.H. thanks the DAAD for financial support. J. Park and A. E. Sakdinawat have kindly contributed the x-ray microdiffraction data.

\section{References}

(1) Gutzow, I.; Schmelzer, J. The Vitreous State; Springer: Berlin, 1995.

(2) Wuttig, M.; Yamada, N. Phase-Change Materials for Rewriteable Data Storage. Nat. Mater. 2007, 6, 824-832.

(3) Wong, H. P.; Raoux, S.; Kim, S.; Liang, J.; Reifenberg, J. P.; Rajendran, B.; Asheghi, M.; Goodson, K. E. Phase Change Memory. Proc. IEEE 2010, 98, 2201-2227. 
(4) Ohta, T. Phase-Change Optical Memory Promotes the DVD Optical Disc. J. Optoelectron. Adv. Mater. 2001, 3, 609 .

(5) Tammann, G. Der Glaszustand; Verlag von Leopold Voss: Leipzig, 1933.

(6) Lencer, D.; Salinga, M.; Wuttig, M. Design Rules for Phase-Change Materials in Data Storage Applications. Adv. Mater. 2011, 23, 2030.

(7) Bruns, G.; Merkelbach, P.; Schlockermann, C.; Salinga, M.; Wuttig, M.; Happ, T. D.; Philipp, J. B.; Kund, M. Nanosecond Switching in GeTe Phase Change Memory Cells. Appl. Phys. Lett. 2009, 95, 3108.

(8) Greer, A. L. Crystal Nucleation and Growth in Metallic Liquids and Glasses. In Amorphous Metals and Semiconductors: Proceedings of an international Workshop; Haasen, P.; Jaffee, R. I., Eds.; Pergamon Press, 1986; pp. 94-194.

(9) Orava, J.; Greer, A. L.; Gholipour, B.; Hewak, D. W.; Smith, C. E. Characterization of Supercooled Liquid Ge2Sb2Te5 and Its Crystallization by Ultrafast-Heating Calorimetry. Nat. Mater. 2012, 11 279-283.

(10) Kalb, J.; Spaepen, F.; Wuttig, M. Atomic Force Microscopy Measurements of Crystal Nucleation and Growth Rates in Thin Films of Amorphous Te Alloys. Appl. Phys. Lett. 2004, 84, 5240.

(11) Salinga, M.; Carria, E.; Kaldenbach, A.; Bornhöfft, M.; Benke, J.; Mayer, J.; Wuttig, M. Measurement of Crystal Growth Velocity in a Melt-Quenched Phase-Change Material. Nat. Commun. 2013, 4, 2371.

(12) Raoux, S.; Cheng, H.-Y.; Caldwell, M. A.; Wong, H.-S. P. Crystallization Times of Ge - Te Phase Change Materials as a Function of Composition. Appl. Phys. Lett. 2009, 95, 071910.

(13) Weidenhof, V.; Friedrich, I.; Ziegler, S.; Wuttig, M. Laser Induced Crystallization of Amorphous Ge2Sb2Te5 Films. J. Appl. Phys. 2001, 89, 3168.

(14) Sebastian, A.; Le Gallo, M.; Krebs, D. Crystal Growth within a Phase Change Memory Cell. Nat. Commun. 2014, 5, 4314.

(15) Jeyasingh, R.; Fong, S. W.; Lee, J.; Li, Z.; Chang, K.-W.; Mantegazza, D.; Asheghi, M.; Goodson, K. E.; Wong, H.-S. P. Ultrafast Characterization of Phase-Change Material Crystallization Properties in the Melt-Quenched Amorphous Phase. Nano Lett. 2014, 14, 3419-3426.

(16) Santala, M. K.; Reed, B. W.; Raoux, S.; Topuria, T.; LaGrange, T.; Campbell, G. H. Irreversible Reactions Studied with Nanosecond Transmission Electron Microscopy Movies: Laser Crystallization of Phase Change Materials. Appl. Phys. Lett. 2013, 102, 174105.

(17) Siegel, J.; Schropp, A.; Solis, J.; Afonso, C. N.; Wuttig, M. Rewritable Phase-Change Optical Recording in Ge2Sb2Te5 Films Induced by Picosecond Laser Pulses. Appl. Phys. Lett. 2004, 84, 2250.

(18) Siegel, J.; Gawelda, W.; Puerto, D.; Dorronsoro, C.; Solis, J.; Afonso, C. N.; de Sande, J. C. G.; Bez, R.; Pirovano, A.; Wiemer, C. Amorphization Dynamics of Ge2Sb2Te5 Films upon Nano- and Femtosecond Laser Pulse Irradiation. J. Appl. Phys. 2008, 103, 023516. 
(19) Shu, M. J.; Chatzakis, I.; Kuo, Y.; Zalden, P.; Lindenberg, A. M. Ultrafast Sub-Threshold Photo-Induced Response in Crystalline and Amorphous GeSbTe Thin Films. Appl. Phys. Lett. 2013, 102, 1903.

(20) Lee, B.-S.; Burr, G. W.; Shelby, R. M.; Raoux, S.; Rettner, C. T.; Bogle, S. N.; Darmawikarta, K.; Bishop, S. G.; Abelson, J. R. Observation of the Role of Subcritical Nuclei in Crystallization of a Glassy Solid. Science 2009, 326, 980-984.

(21) Bessas, D.; Sergueev, I.; Wille, H. C.; Peron, J.; Ebling, D.; Hermann, R. P. Lattice Dynamics in Bi2Te3 and Sb2Te3: Te and Sb Density of Phonon States. Phys. Rev. B 2012, 86, 4301.

(22) Tyrrell; Harris. Diffusion in Liquids; Butterworth, 1984.

(23) Mauro, J. C.; Yue, Y.; Ellison, A. J.; Gupta, P. K.; Allan, D. C. Viscosity of Glass-Forming Liquids. Proc. Natl. Acad. Sci. U. S. A. 2009, 106, 19780-19784.

(24) Sosso, G. C.; Behler, J.; Bernasconi, M. Breakdown of Stokes-Einstein Relation in the Supercooled Liquid State of Phase Change Materials. Phys. Status Solidi B 2012, 249, 1880-1885.

(25) Kelton, K. Crystal Nucleation in Liquids and Glasses. In Solid state physics Vol. 45; Academic Press, 1991; pp. 75-177.

(26) Johannes Andreas Kalb. Crystallization Kinetics in Antimony and Tellurium Alloys Used for Phase Change Recording, Dissertation RWTH Aachen University, 2006.

(27) Matsunaga, T.; Umetani, Y.; Yamada, N. Structural Study of a Ag3.4In3.7Sb76.4Te16.5 Quadruple Compound Utilized for Phase-Change Optical Disks. Phys. Rev. B 2001, 64, 184116.

(28) Lee, B.-S.; Burr, G. W.; Shelby, R. M.; Raoux, S.; Rettner, C. T.; Bogle, S. N.; Darmawikarta, K.; Bishop, S. G.; Abelson, J. R. Observation of the Role of Subcritical Nuclei in Crystallization of a Glassy Solid. Science (80-. ). 2009, 326, 980.

(29) Njoroge, W. K. Phase Change Optical Recording - Preparation And X-Ray Characterization of GeSbTe And AgInSbTe Films, Dissertation RWTH Aachen University, 2001.

(30) Kolmogorov, A. N. Selected Works of A. N. Kolmogorov, Volume II Probability Theory and Mathematical Statistics; Shiryayev, A. N., Ed.; Springer Netherlands, 1992.

(31) Zhang, W.; Ronneberger, I.; Zalden, P.; Xu, M.; Salinga, M.; Wuttig, M.; Mazzarello, R. How Fragility Makes Phase-Change Data Storage Robust: Insights from Ab Initio Simulations. Sci. Rep. 2014, 4, 6529.

(32) Bishop, S. G.; Taylor, P. C.; Mitchell, D. L.; Slack, L. H. Far Infrared and Microwave Conductivity Spectrum of Semiconducting TI2Se·As2Te3 Glass. J. Non. Cryst. Solids 1971, 5, 351-357.

(33) Wang, L.-M.; Angell, C. A.; Richert, R. Fragility and Thermodynamics in Nonpolymeric Glass-Forming Liquids. J. Chem. Phys. 2006, 125, 074505.

(34) Böhmer, R.; Ngai, K. L.; Angel, C. A.; Plazek, D. J. Nonexpontential Relaxations in Strong and Fragile Glass Formers. J. Chem. Phys. 1993, 99, 4201. 
(35) Kalb, J. A.; Wuttig, M.; Spaepen, F. Calorimetric Measurements of Structural Relaxation and Glass Transition Temperatures in Sputtered Films of Amorphous Te Alloys Used for Phase Change Recording. J. Mater. Res. 2007, 22, 748.

(36) Carria, E.; Mio, A. M.; Gibilisco, S.; Miritello, M.; D’Acapito, F.; Grimaldi, M. G.; Rimini, E. Polymorphism of Amorphous Ge2Sb2Te5 Probed by EXAFS and Raman Spectroscopy. Electrochem. Solid-State Lett. 2011, 14, H480.

(37) Moynihan, C. T.; Easteal, A. J.; Wilder, J.; Tucker, J. Dependence of the Glass Transition Temperature on Heating and Cooling Rate. J. Phys. Chem. 1974, 78, 2673.

(38) Mallamace, F.; Branca, C.; Corsaro, C.; Leone, N.; Spooren, J.; Chen, S.-H.; Stanley, H. E. Transport Properties of Glass-Forming Liquids Suggest That Dynamic Crossover Temperature Is as Important as the Glass Transition Temperature. Proc. Natl. Acad. Sci. U. S. A. 2010, 107, 22457-22462.

(39) Ito, K.; Moynihan, C. T.; Angell, C. A. Thermodynamic Determination of Fragility in Liquids and a Fragile-to-Strong Liquid Transition in Water. Nature 1999, 398, 492-495.

(40) Zheludev, N.; Kivshar, Y. From Metamaterials to Metadevices. Nat. Mater. 2012, 11, 917.

(41) Michel, A.-K. U.; Chigrin, D. N.; Maß, T. W. W.; Schönauer, K.; Salinga, M.; Wuttig, M.; Taubner, T. Using Low-Loss Phase-Change Materials for Mid-Infrared Antenna Resonance Tuning. Nano Lett. 2013, 13, 3470.

(42) Cao, T.; Wei, C.; Simpson, R.; Zhang, L.; Cryan, M. Fast Tuning of Double Fano Resonance Using A Phase-Change Metamaterial Under Low Power Intensity. Sci. Rep. 2014, 4, 4463.

(43) Wright, C. D.; Hosseini, P.; Diosdado, J. a. V. Beyond von-Neumann Computing with Nanoscale Phase-Change Memory Devices. Adv. Funct. Mater. 2013, 23, 2248-2254.

(44) Jóvári, P.; Kaban, I.; Steiner, J.; Beuneu, B.; Schöps, A.; Webb, A. "Wrong Bonds" in Sputtered Amorphous Ge2Sb2Te5. J. Phys. Condens. Matter 2007, 19, 335212. 